\title{
A Critical Review of Classification of Organisations in Relation to the Voluntary Implementation of Environmental Management Systems
}

\author{
Paul A. Stevens, William J. Batty, Phil J. Longhurst, Gillian H. Drew ${ }^{1}$ \\ Centre for Energy and Resource Technology, Department of Environmental Science and \\ Technology, School of Applied Sciences, Building 40, Cranfield University, Cranfield, MK43 \\ $O A L, U K$
}

\section{Highlights:}

- We identify the relationship between environmental management and classification

- $\quad$ Existing attempts to classify organisations are critically reviewed

- No practical application exists or the classes proposed are inadequate

- A new means of categorising organisations that is "fit for purpose" is

provided

\footnotetext{
${ }^{1}$ Corresponding author: Gillian Drew; g.h.drew@cranfield.ac.uk; Tel: + 44 (0)1234 750111; Fax. $+44(0) 123475167$
} 


\title{
A Critical Review of Classification of Organisations in Relation to the Voluntary
} Implementation of Environmental Management Systems

\author{
Paul A. Stevens, William J. Batty, Phil J. Longhurst, Gillian H. Drew ${ }^{1}$ \\ Centre for Energy and Resource Technology, Department of Environmental Science and \\ Technology, School of Applied Sciences, Building 40, Cranfield University, Cranfield, MK43 \\ $O A L, U K$
}

Keywords: Environmental Management, ISO 14001, Classification, Classes, Taxonomies

\begin{abstract}
:
The need and ability of an organisation to manage and control its impact on the environment has been hotly debated in recent times. However, the uptake of certificated environmental management systems (EMS), specifically BS EN ISO 14001 (ISO 14001) (British Standards Institution, 2004), is becoming more prevalent, even though evidence of the individual benefits are less clear. Furthermore, reports are often limited and anecdotal in their discussion of the true barriers that organisations experience during the certification and management of their EMS. Presently organisations are commonly classified simply according to size and the barriers they experience when implementing an EMS successfully. This system of classification is not sufficient to understand the multifaceted environments within which modern organisations operate.

This paper reviews existing classification methodologies relevant to environmental management so as to determine whether opportunities exist for their practical application in this sector. It begins with an introduction to EMS and existing discussions regarding implementation is provided before a more detailed consideration of organisational size, the integration and development of environmental management within an organisation, then cladistics and quality management systems (QMS) are reviewed as potential opportunities for classification. This shows that whilst numerous methods are available, none function beyond the theoretical, or that the classes provided restrain the description of the complex tasks.
\end{abstract}

Central to differences faced by organisations are insights to the true hurdles that each experience when implementing an EMS. It is shown here how the manipulation of techniques from the more mature field of Energy Management may offer a direction for the

\footnotetext{
${ }^{1}$ Corresponding author: Gillian Drew; g.h.drew@cranfield.ac.uk; Tel: + 44 (0)1234 750111; Fax. $+44(0) 123475167$
} 
development of robust classes. A valuable outcome is that these methods produce implementation and management of their EMS.

\section{Introduction}

For any company the most important factors for survival are to remain profitable, competitive and to increase market share. However, as organisations become ever more accountable for their social and environmental impact (Porter and Kramer, 2006), they must treat their operational impact on the local and wider environment as a fundamental aspect of policy design and demonstrate engagement in this through better environmental management (Aiyub et al., 2009).

Environmental management has historically taken the form of command and control regulations stemming from broad principles or objectives introduced at international level, which have become ratified through international treaties or conventions (Brady, 2005). Whilst regulatory controls have been attributed with achieving substantial improvements in the reduction of industrial pollution they have also experienced criticism for being inflexible and not cost-effective. Consequently, the direction of control mechanisms has subsequently moved towards levies and tradable permits, but these have also been found to be inadequate for dealing with the complexities of the emissions of multiple pollutants (Ziegler and Nogareda, 2009). During recent decades these inadequacies have prompted increased development and diffusion of environmental management through the agency of private or non-governmental organisations; encouraging firms to reach beyond legislative compliance (Delmas and Toffel, 2008).

Considerable, although not uniform, agreement exists that private instruments are both beneficial and have the ability to run concurrently with existing command and control policies (Alberini and Segerson, 2002;Arimura et al., 2008). Darnall (2003) suggests a combination of institutional pressures and internal competencies as explanations for firms' participation in voluntary environmental programmes, highlighting; regulatory pressure, social pressures, continual improvement capabilities and capital expenditure as motivations for the early adoption of certified Environmental Management Systems (EMS).

There has been considerable development of voluntary models for environmental management, those which go beyond legislative requirements and the pressure to undertake their implementation (Delmas and Montiel, 2008). By far the most commonly used, and so discussed, of these instruments are BS EN ISO 14001:2004 (ISO 14001) ) in the UK and the Environmental Management and Audit Scheme (EMAS) in Europe, with 
substantial levels of research attributed to their application (Delmas and Montiel, 2008;Giles, 2008; Hillary, 2004)

Many authors have discussed the perceived barriers to EMS implementation within firms, citing; the possibility of negative publicity and uncertainty over future regulatory controls (Delmas, 2000;Hillary, 2004;Fresner, 2004;Yin and Schmeidler, 2009). Additionally, and specifically in smaller firms; a lack of the required resources, including both finance and time, lack of rewards and added bureaucracy are stated as barriers to the uptake of EMS (Hillary, 2004). However, such arguments are relatively undeveloped with discussion largely based on anecdotal evidence and small scale case studies with results being used to draw general conclusions across the diverse and multifaceted environments that companies operate within.

Hunt and Auster (1990) highlighted the impact of companies' associations with negative environmental impacts, of which they include loss of competitive advantage, strained relationships with suppliers and vendors, extensive cost and loss of public image. They continue to surmise that a solution to these issues cannot be reached through a 'band aid' approach to environmental management, rather that if true and sustainable benefits are to be made then investment should be made in resource reduction management programmes.

However, even with the growth in legislative and voluntary controls, companies approach this growing challenge with diverse perspectives on the values and benefits of compliance or strategies that move beyond compliance. Companies, often of similar circumstance, vary; from those who support environmentally sound initiatives to those that avoid compliance even with existing legislation (Bansal and Roth, 2000). Paulraj (2009) discusses the need to understand the differing motives for environmental strategies and their correlation with environmental behaviour and how this often is only undertaken through 'company level' focus. Therefore, a better understanding is required of the criteria or circumstances that cause companies to display particular behavioural patterns in response to the notion of EMS. Consequently, the ability to be able to comprehensively understand the behaviours of companies in terms of business culture and structure parameters will enable the cultivation of mechanisms that support environmentally sustainable practices and help to avoid the reported barriers faced during their implementation and management within the wider company environment.

The objective of this paper is to investigate opportunities and limitations for the categorisation of organisations. It is argued that this will further understanding of the factors that influence EMS implementation, as opposed to reiterating existing discussions on EMS. More appropriate categorisation should enable specific motivations and barriers, associated 
with EMS, to be attributed to applicable organisations. This should allow for more resource efficient promotion, implementation and management of an individual EMS.

\section{Existing Methods of Organisational Classification}

It is proposed that, to better understand the barriers to EMS implementation for the multitude of participants, broader methods of classifying companies should be employed. McKelvey (1978) offers benefits of "General Classification", as a methodology that groups objects by all their individual attributes, to include: 1) Strengthening of scientific understanding that enables homogeneous groups to be defined and subsequent hypotheses to be tested, 2) The ability to provide more comprehensive information retrieval, 3) Providing a conceptual framework capable of understanding and communicating the intrinsic diversity existing across companies, and 4) Applying classification schemes to other areas of a company including behaviour, development and practical management.

However, Goodall's (1954) paper entitled "Vegetational classification and Vegetational continua", cited in McCarthy (1995) suggests attempts at classification can result in nothing more than a waste of valuable scientific time, relating the problem to one of confusion through an inadequate understanding of the multiple taxonomical methodologies available as opposed to a fundamental desire to classify. McKelvey (1978) distinguishes taxonomy from classification as a process that not only identifies and assigns characteristics to recognised classes, but also provides a theory for the development of defined differences. Therefore if taxonomical reference is to be made in application to an understanding of the classification of firms, it is imperative that a clear and unambiguous understanding of the organisational behaviour be achieved. Furthermore, these must incorporate time series studies that of specific attributes that encompass the complexities of modern organisational culture.

\subsection{Classification through Organisational Size}

In an effort to better understand the barriers to EMS implementation, size dependant classes have been proposed. However, the division of firms is often as basic as; "Small to Medium Sized Enterprises" (SME's) and non SME's (Williams et al., 2000;Koroljova and Voronova, 2007; Heras and Arana, 2010). However, within the UK, the split between SME and non SME is shown to be $99 \%$ and $1 \%$ respectively (Department for Business Innovation and Skills, 2010).

With $99 \%$ of companies within the UK falling within the category of SME, it seems reasonable to assume that, although some similarities may exist between companies within the SME and non-SME classes, there will also be considerable differences and variations within each category in terms of the types of: business activity, organisational culture, 
resource availability, staff skills and challenges faced. Consequently, different requirements and expectations will be placed on the barriers faced when achieving implementation of an EMS, particularly related to the availability of different staff abilities and skills. The potential ambiguity of results obtained from the application of a limited choice of classes such as SME or non-SME, suggests that a 'one size fits all' option for effective and efficient environmental management, within such broadly define classes is neither practical nor useful. Consequently, a more discriminating classification of firms is required to support the diversities of activities, with which each company is involved, as well as the skills, qualification and motivation exhibited by the stakeholders investing in environmental management initiatives.

With such a high percentage of UK industries classified as SME's, a correlation between their financial turnovers and environmental impacts might seem inevitable, nevertheless, the complexities and the levels of correlation are still ardently debated (Yin and Schmeidler, 2009;Giles, 2008;Rowland-Jones et al., 2005). It seems reasonable to assume that an industry and commerce category that accounts for $99.8 \%$ of all the enterprises in the European Union, $47 \%$ of total turnover and $48 \%$ of employment would also account for a substantial percentage of the overall environmental impact of firms. Pimenova (2004) offered an estimation of the environmental impact attributable to SME's as high as $70 \%$ of the total. Although, with reference to research undertaken in six European Union countries, Pimenova (2004) concludes that the total is more likely to be in the region of $50 \%$ bringing it in line with the relative proportions of turnover and employment of SME's. The argument for the initially higher percentage estimation was related to the impacts arising from the externalities of firms' activities and therefore not included in calculation methodologies (Kenny and Gray, 2009; Carbon Trust, 2008;DECC, 2009). It could also be argued that office floor area usage may be less dense for SME's resulting in higher environmental impacts per person in this sector. Zorpas (2010) confirms this confusion, highlighting that the heterogeneous nature of SME's makes generalisation about environmental issues very difficult.

Through a more discriminating classification of the sector more specific barriers and motivations might be discerned and understood and therefore more fitting approaches taken in both the marketing and application of EMS. Hillary (2004) highlights the inherent heterogeneity prevalent in a single category as broad as SME's thus highlighting a disparity, and possible deficit, in the knowledge required to support the mounting enthusiasm towards sustainability. Hillary (2004) concluded that future research in this field required further subdivision of the category of SME by either; size or by industry sector. However, no explanations for these specific choices of classes are discussed or reasoning for their preeminence provided. 
Zorpas (2010) expanded on the work of Hillary (2004) by undertaking a detailed analysis of Union's (2005) SME definition. The research endeavours to distinguish the different needs, wants and stimuli for EMS implementation in SME's and the subcategory of VSME. Zorpas (2010) hypothesised that VSME's often provided mono-service or product offerings and that they were disproportionally affected, in a negative manner, by economic pressures. Additionally, it was suggested that the responses of VSME to new requirements of the market were restricted through little access to further training; non participate in knowledge transfer or developing cross firm federation whilst producing high levels of waste (Zorpas et al., 2008). However, contradictory research suggests that SME's experience distinct advantages over non-SME's when implementing green initiatives including less bureaucracy, quicker response time and efficient internal communication (Van Hemel and Cramer, 2002). This debate highlights the inefficiencies of organisational classes such as size for categorising firms. If the classes developed are unrepresentative of actual activities the resulting classification will also be unrepresentative of the barriers and benefits experienced by participating firms.

Separate research within this field has approached the understanding of SME's and EMS implementation in terms of its integration within an organisation, by focusing on the motivations driving the process and attempting to offer a classification methodology based on this (Paulraj, 2009;Jabbour and Santos, 2006).

\subsection{Classification Based on Stages of Integration, Development and Evolution}

Jabbour and Santos (2006) discussed environmental management classification as a taxonomical study that is not size dependant but rather related to the achieving of a "stage" within an evolutionary process dependant on the level to which environmental strategies and actions are integrated within a particular organisation. Jabbour and Santos (2006) summarised this research and propose taxon comprising of three evolutionary stages of environmental management; (i) functional specialisation, (ii) internal integration and (iii) external (or strategic) integration. Jabbour and Santos (2006) suggested that each taxon draws together several criteria for defining the environmental management maturity that may be present within a firm and proposed that the level of maturity found within firms ultimately correlates with a firm's overall organisational configuration. However, as with the classification of companies by size; SME, small enterprise (SE) and very small enterprise (VSE) this taxonomy only provides three options within which to place all firms and with very broad criteria for inclusion. It must be questioned whether such broad classes (taxa) would be able to provide sufficiently clear discrimination between participants. 
Jabbour and Santos (2006) bases much of the conclusions drawn from their study on an investigation of 37 firms that had achieved certification to ISO 14001, implying that all of these firms had a degree of understanding and commitment to improvements in environmental management. However, Jabbour and Santos (2006) states that these firms still demonstrated discrete behaviours, suggesting that these could be attributed to specific organisational contexts. This heterogeneity is a phenomenon that has been neglected in current literature and Yin and Schmeidler (2009) state that, due to the 'standardised' nature of management systems they are often linked with a perception of homogeneity that encourages unrealistic conclusions being drawn as to the similarity of the processes or structures of one organization to those of another (isomorphism). Furthermore, it raises the question as to whether the scope of the conclusions reached by Jabbour and Santos (2006), for companies that have achieved ISO 14001 certification, can be expanded to include companies that have yet to, or have no intention of, acquiring certification to ISO 14001. Including such companies within a limited "evolutionary" matrix may impact on the usefulness of the proposed taxonomy, especially when existing variations in behavioural patterns are taken into account. This implies a need for a wider research base and for a greater number of relevant classes (taxa) to be considered. Paulraj (2009) strengthens this argument through concluding that the research undertaken to date had been modest and based on "a parsimonious set of motivational indicators". Therefore, Paulraj (2009) determines that future research is required that not only refines and strengthens the identified constructs, but also expands both the theoretical and empirical bases of future research through the use of additional motivational indicators and a broader set of descriptive variables.

\subsection{Classification through the use of Cladistics}

It has been suggested that an organisation's approach to EMS implementation can have a direct impact on the internal development of the scheme (Yin and Schmeidler, 2009) and overly simplistic classification may ignore the heterogeneous nature of organisations (Hillary, 2004). Therefore a more comprehensive process of defining classes that incorporates behavioural aspects of an organisation should be established in relation to environmental management systems. McKelvey (1978) suggests that systematics have the potential to dramatically improve the understanding of organisations. This is where the complete system is considered in order to understand differences and their relationships with the wider environment. Subsequently, this process can provide a meaningful method of classification. He cites significant similarities between organisations and organisms. Systematics include three main components: (i) Taxonomy - the construction of defined organisational distinctions; (ii) evolution - the mapping of the ancestry of biological the distinctions and (iii) 
classification - the implementation of processes that enable organisational forms to be placed into classes. However, McKelvey (1978) stated that organisational systematics required both numerical taxonomic and phyletic theories of classification, which are explained in detail in McKelvey (1978).

However, McKelvey (1978) also suggests that focusing on only one or two attributes of an organisation may offer high predictive validity but only in relation to the prescribed areas of organisational behaviour, entitling this "Special Classification". Therefore, special classification is only beneficial if the attribute(s) included are the point of focus and offers poor mechanisms for retrieval and understanding of the relationships between wider organisational behaviours. That is to say where a functional study uses taxa based on size it may be difficult to transpose this knowledge to taxa relating to age, bureaucracy or complexity within organisations (McKelvey, 1978).

McCarthy (1995) incorporated the work of McKelvey (1978) to consider the application of the concepts of organisational systematics and biological taxonomy in an attempt to classify firms within the manufacturing sector. McCarthy (1995) discusses the notions of numerical, essentialistic, nominalistic and cladistic theories as methodologies for the development of taxa to be used for such classifications. Cladistics, although originating in the classification of languages and then migrating into biological evolutional theory, has become embedded in attempts to make classifications within the manufacturing sector (Leseure, 2002). Leseure (2002) suggested that cladistics was initially deemed "lowbrow" by evolutionary biologists in comparison to phenetics, a quantitative classification technique based on the use of cluster analysis. Cladistics, a systematisation technique, allows "specimens" to be grouped into systems and because of this "cladistics can be applied to study the history of any evolving system: the evolution of management ideas, of beliefs, of products, of technologies, etc." (Leseure, 2002). McCarthy et al (2000) suggested that cladistics is the dominant approach in biology and therefore the most suitable tool for classification of manufacturing companies. Leseure (2002), supported this conclusion, suggesting that cladistics, rather than phenetics was the most applicable tool within this field.

McCarthy (1995) shows that cladistics, through their wider application, have developed a set of rules and principles. These rules are concerned with operational principles, such as branching and labelling. It is suggested that these rules are transferable to operational principles of other industries and systems, specifically manufacturing. However McCarthy (1995), although offering a schematic of a dendrogram, does this only in an explanatory context, as opposed to one capable of practical application. McCarthy (1995) concludes that a relatively precise, stable, enduring and universal classification could be achieved and that 
this would significantly improve understanding of, in this instance, manufacturing company accuracy and value of predictions which would subsequently bring about 'ideal' category specific models and solutions.

However, although the practice of cladistics enables multiple taxa and the resulting expansion of classes beyond those found when using industry size and type alone, it does have limitations and is not without detractors. The processes have been described as posthoc with limitations specifically related to future decision making processes or evolutionary trajectories such as the implementation of innovative technologies and advanced systems (Baldwin et al., 2005). In this context, post-hoc analyses are concerned with finding patterns and/or relationships between subgroups of sampled populations that would otherwise remain undetected were the investigators to have to rely strictly upon a-priori statistical methods. Such analyses form a valuable collection of tools which allow exploratory research greater freedom. McCarthy (1995) highlighted that the essential attributes of taxa should be appropriate, with reference in his paper to the manufacturing sector. In this instance the development of taxa had been made in reference to the classification of firms and considered a number of factors which must be:

- mutually exclusive - taxa must not allow for a firm's inclusion in more than one category:

- Internally homogenous - certain discrete behaviour must be excluded during classification:

- Collectively exhaustive - taxa provided must ensure complete inclusion of all participating firms:

- Stable - further empirical tests of firms should not affect the predetermined taxa:

- Relevant in terms of naming - to ensure effective communication naming of taxa should be based on common academic and business language (McCarthy, 1995).

Furthermore, Leseure (2002), McCarthy (1995) and McCarthy et al (2000), although providing eloquent arguments supporting the benefits of cladistics, did so from a very fundamental stand point. They do not provide either empirical evidence of application or the methodology used to achieve the conclusions drawn. Furthermore, no empirical evidence was provided to support their theory that classification can aid a decision making process was lacking. The link between theory and practical application of this argument is missing, as is the inclusion of constraints for the construction of robust taxa. It is implied but not 
explicitly explained by the authors, that cladistics can provide a classification system based (1998), as providing "relative statements of relationship" rather than being capable of "explicitly hypothesising ancestor-descendent relationships" (Lipscomb, 1998).

\subsection{Classification Related to the Concepts of Quality Management}

A further opportunity to expand the classes used when categorising firms in relation to their environmental management is their integration with practices more usually discussed within the context of quality management. This link has been keenly discussed in relation to ISO 14001 and the quality management system (QMS) BS EN ISO 9001:2000/2008 (ISO 9001) (Zeng et al., 2005;Castka and Balzarova, 2008;Tarí and Molina-Azorín, 2010). Zeng et al (2005) showed that the term integration itself has multiple definitions and that although not necessarily contradictory these terms do focus on specific characteristics that therefore alter the meaning of the word in discrete contexts.

Gavin (1991) focuses on integration in relation to the ability to offer synchronization and unity within an organisation, whereas MacGregor Associates (1996) see integration as a singular, top level, standard capable of supporting modular attachments for specific requirements. This complicates the work of authors such as Jabbour and Santos (2006), who provide a classification process based on 'integration' to evaluate environmental management and to develop a supporting classification of companies. Furthermore the methods foundation is in reviews of existing research as opposed to empirical trials that would suggest an opportunity for practical application. Additionally, due to the very limited classes offered (3) the process does not promote integration as a classification process over other discriminatory behaviours.

This is not to say that the environmental management and its integration with quality management cannot support the development of classes that benefit the understanding, and subsequent ability to offer a beneficial service to SME's. Using the requirements of ISO 14001 and ISO 9001 (Annex B of ISO 14001 and Annex A of ISO 9001) (British Standards Institution, 2004;British Standards Institution, 2008), the internationally recognised and extensively adopted EMS and QMS (Zeng et al., 2005) highlight that opportunities for integration are clear with every specific requirement of ISO 14001 having a direct link to the similar requirements within ISO 9001.

This review of ISO 14001 Annex B (British Standards Institution, 2004) also highlights another simplification in the available classes and may go some way to explain the discrete behaviours shown by companies attempting to attain certification. Within most of the relevant literature, ISO 14001 is discussed as a single standard and not in terms of its 
multiple requirements to which firms must comply for certified compliance (Hillary, 2004;Zorpas, 2010;Giles, 2008;Yin and Schmeidler, 2009). It has been clearly shown that different firms approach ISO 14001 implementation and management differently (Yin and Schmeidler, 2009; Hillary, 2004;Fresner, 2004), therefore it can be concluded that firms will approach and integrate individual challenges discreetly too. Consequently a more comprehensive classification methodology might be able to incorporate these behavioural attributes more readily.

\section{Future Opportunities for Classification}

Lessons in classification may also be learnt through the investigation of energy management, a longer studied and more mature research field that offers considerable similarities with the current interests in environmental management. The use of ISO standards in applicable disciplines clearly shows a significant link in the requirements of both energy and environmental management (British Standards Institution, 2011). It may then be concluded that lessons already learnt in the field of energy management may be both applicable and beneficial in avoiding wasted effort through the "reinvention of the wheel", in research into the uptake of EMS.

Instead of attempting to channel firms and their discreet attributes into pre-determined and confining classes reliant on perceived similarities, a more logical process would be to develop an understanding of both the similarities and differences present. Fawkes (1978) proposed aspects of company structure, resource base and character that potentially allow a move away from a limited approach to classification by offering the "Seven S Approach". In this approach he considered the description of company organisation and culture in terms of 7 classes as follows:

- Superordinate goals: the guiding concepts instilled by an organisation in its members.

- Strategy: the process by which an organisation allocates its finite resources to achieve desired outcomes.

- Structure: the characteristics of the organisation's structure.

- Systems: proceduralised processes.

- Skills: possessed by either individuals, groups or the firm as a whole.

- Style: the behaviour and characteristics of key managers in the implementation of organisational goals as well as the organisational culture. 
Staff: the breakdown of significant employee classes (Fawkes, 1987).

Although the seven S's concept was intended to allow behaviours of companies to be differentiated, this methodology also provides an opportunity to categorise aspects of company behaviour. An example of this is provided when Western and Japanese management styles are compared and their differences highlighted using the seven $\mathrm{S}$ factors (Pascale and Athos, 1982). This work provides a set of classes that allow discreet behavioural patterns to be identified. The Seven S system will allow the inclusion of the previously discussed classification methods (Figure 1), to develop a methodology for studying the behaviours of firms in relation to environmental, energy or quality management requirements.

To move from providing the theoretical basis for research to the development of a practical methodology, the Seven S model requires a structured platform to enable its application. Existing techniques from the energy sector may be able to provide this structure in the form of the well established notion of the Energy Management Matrix (BRECSU). This tool, in its original guise, acted as an effective method of increasing the understanding of a company's energy management philosophy and practice (Ashford, 1993).

The matrix columns deal, individually, with areas pertinent to energy management and the rows offer qualitative descriptions of increasingly sophisticated controls for these. The use of this matrix suggests that through the application of Fawkes (1978) model it may be possible to bridge the existing gap between a theoretical basis for classification and a practical methodology.

\section{Discussion}

Research surrounding EMS and its impact on companies is extensive and clearly highlights a growing pressure on them to incorporate green controls within their operational procedures. The evidence that different firms approach and experience EMS implementation and management differently remains mostly anecdotal and has been derived from research where these aspects were of secondary concern to the purpose of the studies.

Additionally, reported notions concerned with methods of classification, particularly in relation to EMS appear to be either convoluted or overly simplistic in terms of the taxa proposed. Proposed approaches focus on only a small aspect of company behaviours in response to environmental management and so require companies to be 'shoe horned' into classes that subsequently are unable to allow the discreet behaviours they may exhibit to be distinguished. Rather existing research, including that of Hillary (2004), Koroljova and Voronova (2007), Heras and Arana (2010) and Yin and Schmeidler (2009), highlights the 
need to better understand the heterogeneity inherent within smaller enterprises and the capable of converting theory into applicable methods from which conclusions might be drawn.

Previous research has discussed the reasoning for EMS implementation in terms of motivations driving the process, even attempting to categorise firms by this (Paulraj, 2009; Jabbour and Santos, 2006). However, the need for research to encompass the requirements of differing firms and their wish to achieve different outcomes beyond simple compliance to an EMS has mostly been neglected. Different aspects of ISO 14001 are likely to be given different weightings by companies and so resources will be allocated accordingly. This important aspect of company behaviour has not been factored into any of the classification methodologies proposed to date.

Confusion is compounded by the seemingly interchangeable use of the terms classification and taxonomy. If research does not encompass detailed information surrounding organisational evolution and behaviour and is not based on time series studies it is unable to provide taxa. Consequently the term classification is more accurate when discussing existing research.

Potential exists for research into the implementation of EMS to learn from more established areas such as energy management. Concepts such as the seven $\mathrm{S}$ model and energy management matrices may enable more appropriate and discriminating classes to be defined that offer meaningful and applicable methods of classification. To further research in this field a comprehensive and structured understanding of the discrete behaviours exhibited by companies is required to support the development of a more extensive set of classes. It is proposed, that robust classes may be identified that encompass and integrate multiple parameters of service based systems and that these should be used rather than restrictive sets of classes drawn from a limited view of the field of study. It is also necessary to incorporate the diversity prevalent in organisations that may presently portray similar characteristics when existing taxa are employed and with particular reference to EMS allow for the different motivations for pursuing certification.

For some of the more complex methods of classification there has been a lack of practical application. Cladistics, although developed from a sound academic base, has yet to be proven in the context of environmental management. Furthermore, the variety of research combined with the lack of application has lead to a diverse group of methodologies that, in 
turn, create confusion for future research or application and a fundamentally limited understanding of the notion of taxonomy.

To overcome this hurdle it is proposed that consideration is given to research previously carried out under the title of "Energy Management". Energy management, being extensively developed since the late 1970s, offers transferable knowledge, techniques and skills. It has also been shown that there are considerable similarities between a number of standards including quality, energy and environmental management standards BS EN ISO 50001:2011, ISO 9001 and ISO 14001 respectively. As such it may be possible to apply lessons learnt in the context of any one of these standards to organisations that operate certification to the other standards.

Furthermore, Fawkes' (1987) notion of seven classes within which companies may be described when incorporated with attributes such as size and activity type provides a basis for a method of developing taxa that will enable an understanding of both the similarities and differences present in company behaviours as related to certification and application of EMS. Through the use of the proposed model it may be possible to bridge the existing gap between a theoretical basis for classification and a practical methodology.

\section{Conclusions}

It can be seen that there are significant gaps in our current understanding of the heterogeneity of organisational responses to EMS implementation. Discussion to date has often been based on opinion and anecdote as opposed to evidence. Furthermore, it is clear that there is a requirement for a robust process of categorisation that incorporates behavioural, as opposed to standard metrics for organisations. Such an approach would inform EMS implementation where understanding of the internal process can then be achieved. The key conclusions of this work are:

- An inherent lack of understanding exists regarding the true barriers to the practises involved in the certification and implementation of an EMS

. The division of firms is often as simple as Small to Medium Sized Enterprises" (SME's) and non SME's and this does not support the improvement of the certification process

- No clear basis exists currently for the development of relevant classification processes.

. The process of classification is often confused through the use of multiple methodologies and a limited understanding of the notion of taxonomy. 
- The placing of firms in to groups based upon very broad criteria does not necessarily provide clear discrimination between participants.

- Classification methods such as cladistics, although developed from sound academic bases, are yet to prove their value for practical application in relation to EMS.

- Existing methods of classification neglect to encompass the different requirements of firms and their wish to achieve different outcomes beyond simple compliance to an EMS

. The 7S model potentially provides a basis for developing taxa that better support the understanding of both the similarities and differences present in company behaviours as related to certification and application of EMS.

- It is suggested that the 7S model for company organisational behaviours will help to bridge the existing gap between the theoretical basis for classification and a practical methodology. 


\section{References}

AIYUB, K., JAHI, J. M., ARIFIN, K. and AWANG, A. (2009) Environmental performance in small and medium sized enterprises (SMEs) certified to ISO 14001 in the United Kingdom. International Business Management, 3, 7.

ALBERINI, A. and SEGERSON, K. (2002) Assessing Voluntary Programs to Improve Environmental Quality.

ARIMURA, T. H., HIBIKI, A. and KATAYAMA, H. (2008) Is a voluntary approach an effective environmental policy instrument?: A case for environmental management systems. Journal of Environmental Economics and Management, 55, 281-295.

ASHFORD, C. J. (1993) Energy Efficiency: A Managed Resource. Facilities, 11, 24 - 27.

BALDWIN, J., ALLEN, P. M., RIDGWAY, K. and WINDER, B. (2005) Evolutionary systems: Modelling organisational innovation, Innovation. Innovation: Management, Policy \& Practice, 7, 389-401.

BANSAL, P. and ROTH, K. (2000) Why Companies Go Green: A Model of Ecological Responsiveness. The Academy of Management Journal, 43, 19.

BRADY, J. (2005) Environmental Management in Organizations The iema Handbook, Bath, Earthscan.

BRITISH STANDARDS INSTITUTION (2004) BS EN ISO 14001:2004 Environmental Management Systems Requirements with Guidance for use.

BRITISH STANDARDS INSTITUTION (2008) BS EN ISO 9001:2008 Quality Management Systems Requirements. BSI.

BRITISH STANDARDS INSTITUTION (2011) BS ISO 50001:2011 Energy Management System BSI Standards Publication

CARBON TRUST (2008) Carbon Footprinting: An Introduction for Orginsations. Carbon Trust.

CASTKA, P. and BALZAROVA, M. A. (2008) The impact of ISO 9000 and ISO 14000 on standardisation of social responsibility--an inside perspective. International Journal of Production Economics, 113, 74-87.

DECC (2009) UK's National Stratergy for Climate and Energy: Transition to a low Carbon Society. IN CHANGE, D. O. E. A. C. (Ed.).

DELMAS, M. (2000) barriers and incentives to the adoption of ISO 14001 in the United States. uke Environmental Law and Policy Forum Fall, 1, 38.

DELMAS, M. and MONTIEL, I. (2008) The Diffusion of Voluntary International Management Standards: Responsible Care, ISO 9000, and ISO 14001 in the Chemical Industry. Policy Studies Journal.

DELMAS, M. and TOFFEL, M. (2008) Organizational responses to environmental demands: opening the black box. Strategic Management Journal.

DEPARTMENT FOR BUSINESS INNOVATION AND SKILLS. (2010, 09/06/11). "Enterprise Directorate: Small and Medium Enterprise Statistics for the UK and Regions " Retrieved 09/06/11, 2011, from http://stats.bis.gov.uk/ed/sme/.

EUROPEAN COMMISSION (2005) The new SME definition User guide and model declaration.

FAWKES, S. D. (1987) The seven S approach to energy management. Applied Energy, 28, 235-242.

FRESNER, J. (2004) Small and medium sized enterprises and experiences with environmental management. Journal of Cleaner Production, 12, 545-547.

GILES, F. (2008) Assessing the effectiveness of your environmental management system. Environmental Quality Management, 18, 1-6.

HERAS, I. and ARANA, G. (2010) Alternative models for environmental management in SMEs: the case of Ekoscan vs. ISO 14001. Journal of Cleaner Production, 18, 726-735.

HILLARY, R. (2004) Environmental management systems and the smaller enterprise. SME's and Experiences with Environmental Management Systems, 12, 561-569. 
JABBOUR, C. J. C. and SANTOS, F. C. A. (2006) The evolution of environmental management within organizations: Toward a common taxonomy. Environmental Quality Management, 16, 43-59.

KENNY, T. and GRAY, N. F. (2009) Comparative performance of six carbon footprint models for use in Ireland. Environmental Impact Assessment Review.

KOROLJOVA, A. and VORONOVA, V. (2007) Eco-mapping as a basis for environmental management systems integration at small and medium enterprises. Management of Environmental Quality: An International Journal, 18, 542-555.

LESEURE, M. J. (2002) Cladistics as historiography: part I - introduction to cladistics. Management Decision, 40, 486-496.

MCCARTHY (1995) Manufacturing classification: Lessons from organizational systematics and biological taxonomy. Integrated Manufacturing Systems, 6, 37-48.

PASCALE, R. T. and ATHOS, A. G. (1982) The Art of Japanese Management, London, Penguin.

PAULRAJ, A. (2009) Environmental motivations: a classification scheme and its impact on environmental strategies and practices. Business Strategy \& the Environment (John Wiley \& Sons, Inc), 18, 453-468.

PORTER, M. E. and KRAMER, M. R. (2006) Strategy \& Society: The Link Between Competitive Advantage and Corporate Social Responsibility. Harvard Business Review, 84, 78-92.

ROWLAND-JONES, R., PRYDE, M. and CRESSER, M. (2005) An evaluation of current environmental management systems as indicators of environmental performance. Management of Environmental Quality, 16, 211.

TARÍ, J. J. and MOLINA-AZORÍN, J. F. (2010) Integration of quality management and environmental management systems similarities and the role of the EFQM model. TQM Journal, 22, 687-701.

VAN HEMEL, C. and CRAMER, J. (2002) Barriers and stimuli for ecodesign in SMEs. Journal of Cleaner Production, 10, 439-453.

WILLIAMS, H., VAN HOOYDONK, A., DINGLE, P. and ANNANDALE, D. (2000) Developing Tailored Environmental Management Systems for Small Businesses. Eco-Management \& Auditing (Wiley), 7, 106-113.

YIN, H. and SCHMEIDLER, P. J. (2009) Why do standardized ISO 14001 environmental management systems lead to heterogeneous environmental outcomes? Business Strategy \& the Environment (John Wiley \& Sons, Inc).

ZENG, S. X., TIAN, P. and SHI, J. J. (2005) Implementing integration of ISO 9001 and ISO 14001 for construction. Managerial Auditing Journal, 20, 394-407.

ZIEGLER, A. and NOGAREDA, J. S. (2009) Environmental management systems and technological environmental innovations: Exploring the causal relationship. Research Policy, 38, 885-893.

ZORPAS, A. (2010) Environmental management systems as sustainable tools in the way of life for the SMEs and VSMEs. Bioresource technology, 101, 1544-1557.

ZORPAS, A. A., TSARTAS, P., ARISTIDIS, G. and THEOHAROUS, O. (2008) Mediterranean standard for sustainable tourism (MESST) - general requirements, objectives and the philosophy of MESST. Sustainable Tourism, Wessex Institute of Technology, 3. 
Colour Figure for Web only:

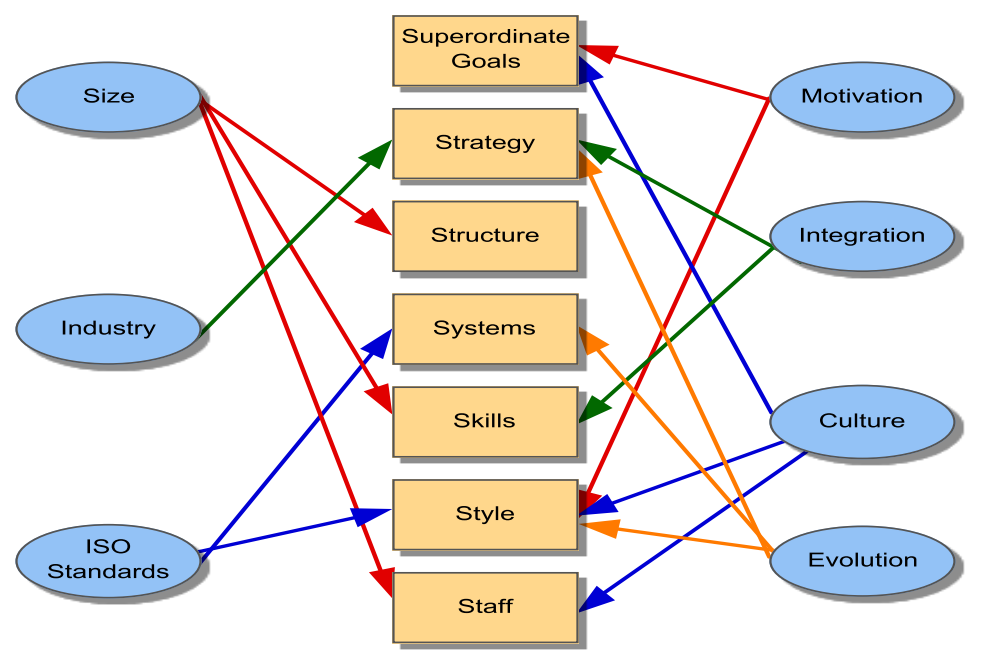

Figure 1: The Seven S Model and the Correlation with Discussed Categories

Black and White Figure for Printing:

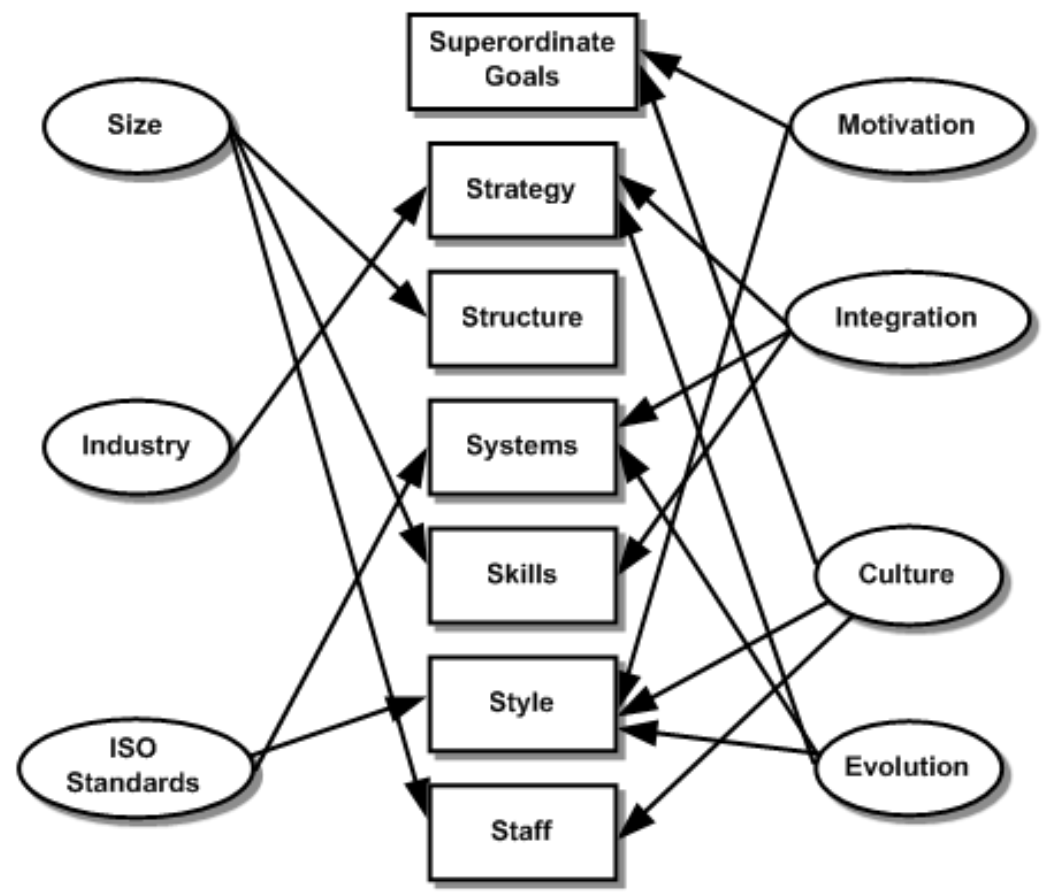

Figure 1: The Seven S Model and the Correlation with Discussed Catogries 